Published in final edited form as:

Expert Rev Anticancer Ther. 2018 August ; 18(8): 793-803. doi:10.1080/14737140.2018.1489245.

\title{
Hypofractionated radiation treatment in the management of breast cancer
}

\author{
Apar Gupta ${ }^{1}$, Nisha Ohri ${ }^{1}$, and Bruce G. Haffty ${ }^{1}$ \\ ${ }^{1}$ Department of Radiation Oncology, Rutgers Cancer Institute of New Jersey, 195 Little Albany St., \\ New Brunswick, NJ 08903, USA
}

\begin{abstract}
Introduction: The standard treatment for early-stage breast cancer is breast conservation therapy, consisting of breast conserving surgery followed by adjuvant radiation treatment (RT). Conventionally-fractionated whole breast irradiation (CF-WBI) has been the standard RT regimen, but recently shorter courses of hypofractionated whole breast irradiation (HF-WBI) have been advocated for patient convenience and reduction in healthcare costs and resources.
\end{abstract}

Areas covered: This review covers the major randomized European and Canadian trials comparing HF-WBI to CF-WBI with long-term follow-up, as well as additional recently closed randomized trials that further seek to define the applicability of HF-WBI in clinical practice. Randomized data is summarized in terms of clinical utility and for a variety of clinical applications. Recently published consensus guidelines and practical implementation of HF-WBI including its broader effect on the healthcare system are reviewed. Finally, an assessment of the emerging evidence in support of hypofractionation for locally advanced disease is presented.

Expert commentary: HF-WBI has replaced CF-WBI as the accepted standard of care in most women with early-stage breast cancer who do not require regional nodal irradiation. Early data supports the continued study of hypofractionation in the locally advanced setting, however broad adoption awaits longer follow-up and additional data from ongoing clinical trials.

\section{Keywords}

breast cancer; breast conservation therapy; conventional fractionation; hypofractionation; whole breast irradiation; radiation treatment

\footnotetext{
*Corresponding author: Bruce G. Haffty, Chief of Staff, Rutgers Cancer Institute of New Jersey, Professor and Chair, Dept of Radiation Oncology, Robert Wood Johnson and New Jersey Medical School, Rutgers, The State University of New Jersey, 195 Little Albany St., New Brunswick, NJ 08903, hafftybg@ @inj.rutgers.edu, Phone: (732) 235-5203, Fax: (732) 253-3953.

Declaration of interest

The authors have no other relevant affiliations or financial involvement with any organization or entity with a financial interest in or financial conflict with the subject matter or materials discussed in the manuscript apart from those disclosed.

Reviewer disclosures

Peer reviewers on this manuscript have no relevant financial or other relationships to disclose.
} 


\section{Introduction}

Breast cancer is the most commonly diagnosed cancer in the world among women [1]. The standard treatment for early-stage breast cancer is breast conservation therapy (BCT), which consists of breast conserving surgery (BCS) followed by adjuvant radiation treatment (RT), with or without adjuvant systemic agents. Multiple large randomized trials of invasive breast cancer over the past half-century have demonstrated that RT significantly reduces locoregional recurrence after BCS, resulting in high rates of breast preservation and excellent survival [2-5]. The Early Breast Cancer Trialists' Collaborative Group (EBCTCG) meta-analysis of 10,801 women with invasive breast cancer in 17 randomized trials found that adjuvant RT after BCS reduced 10-year risk of recurrence from $35.0 \%$ to $19.3 \%$ and 15 year risk of mortality from $25.2 \%$ to $21.4 \%$ [6].

Adjuvant RT for early-stage breast cancer has traditionally utilized 45-50 Gray (Gy) in 1.8-2.0 Gy fractions to the whole breast (with or without an additional tumor bed boost) over the course of five to seven weeks, known as conventionally-fractionated whole breast irradiation (CF-WBI). Historically it was believed that small fraction sizes delivered over a longer time period were necessary for normal tissue sparing. However, in the early 1990s, it was found that breast tumors were equally sensitive to fraction size as late-reacting normal tissues, suggesting that prolonged treatment time does not preferentially spare normal tissues. These findings paved the way for hypofractionated whole breast irradiation (HFWBI), which consists of fraction sizes greater than $2 \mathrm{~Gy}$, delivered to the whole breast (with or without an additional tumor bed boost), to a total dose that is radiobiologically equivalent to a CF-WBI regimen.

The general intent of HF-WBI is to reduce treatment time by reducing the number of individual fractions, creating courses that are shorter and more convenient for patients. Benefits of shorter courses also raise the prospect of increasing use of BCT and access to care while reducing healthcare costs. In this article. we review the randomized data supporting HF-WBI and its clinical use primarily in the management of early-stage breast cancer. We also assess the emerging application of hypofractionation in the locally advanced setting. We do not cover the separate topic of partial breast irradiation for which other comprehensive reviews exist.

\section{Major Randomized Studies Comparing HF-WBI to CF-WBI}

Four major randomized, prospective phase III clinical trials compared HF-WBI to CF-WBI and assessed tumor control outcomes with long-term follow-up, summarized in Table 1.

The first major randomized trial was initiated in 1986 at Royal Marsden Hospital and Gloucestershire Oncology Centre (RMH/GOC) in the United Kingdom (UK) [7,8]. Eligibility criteria included T1-3 N0-1 M0 invasive breast cancers in patients less than 75 years of age who underwent BCS with macroscopically negative margins. Between 1986 and 1998, 1,410 patients were randomized to one of three arms that were all delivered over five weeks: CF-WBI (50 Gy in 25 fractions) or two HF-WBI arms (42.9 Gy in 13 fractions or $39 \mathrm{~Gy}$ in 13 fractions, each delivered every other day). Patients were further randomized 
to a boost of 14 Gy in 7 fractions versus no boost until 1994, after which an elective nonrandomized boost was offered. Thirty percent of patients were less than 50 years of age, $14 \%$ received chemotherapy, $21 \%$ received RNI, and $75 \%$ received a boost. At 10 years, local relapse was $12.1 \%$ in the $50 \mathrm{~Gy}$ arm, $9.6 \%$ in the $42.9 \mathrm{~Gy}$ arm, and $14.8 \%$ in the $39 \mathrm{~Gy}$ arm. While local recurrence between the two HF-WBI arms was significantly different $(\mathrm{p}=0.027$ ), neither HF-WBI arm significantly differed from the control CF-WBI arm. Distant relapse and survival data have not been reported. At 10 years, freedom from marked change in photographic breast appearance was $90.2 \%$ in the $50 \mathrm{~Gy} \mathrm{arm}, 84.4 \%$ in the $42.9 \mathrm{~Gy}$ arm, and $93.4 \%$ in the $39 \mathrm{~Gy}$ arm $(\mathrm{p}<0.001)$. Similarly, there were significant differences in clinical assessment of breast cosmesis, shrinkage, distortion, edema, induration, telangiectasias, and shoulder stiffness, with generally the best performance in the $39 \mathrm{~Gy}$ arm and the worst performance in the $42.9 \mathrm{~Gy}$ arm.

In Canada, the Ontario Clinical Oncology Group (OCOG) initiated a randomized HF-WBI versus CF-WBI trial in 1993 [9,10]. Eligibility criteria included T1-2 N0 M0 invasive cancers in patients who underwent BCS with negative margins on ink. An additional criterion included maximum width of breast tissue $\leq 25 \mathrm{~cm}$ at the central axis. Unlike the RMH/GOC and later START trials, mastectomy, RNI, and boost were not allowed. Between 1993 and 1996, 1,234 patients were randomized to CF-WBI (50 Gy in 25 fractions delivered over five weeks) or HF-WBI (42.56 Gy in 16 fractions delivered over 3.2 weeks). Twentysix percent of patients were less than 50 years of age and $11 \%$ received chemotherapy. At 10 years, local relapse was $7.5 \%$ in the $50 \mathrm{~Gy}$ arm and $7.4 \%$ in the $42.56 \mathrm{~Gy}$ arm, which were not significantly different. Overall survival was also not significantly different $(84.4 \%$ vs $84.6 \%$ ). At 10 years, there was no difference in cosmetic outcome, with $71.3 \%$ and $69.8 \%$ of patients reporting a good or excellent cosmetic outcome in the CF-WBI and HF-WBI arms, respectively. There was similarly no difference in late skin or subcutaneous tissue toxicity.

Given the slightly higher rate of late normal tissue effects in the $42.9 \mathrm{~Gy}$ arm in the RMC/GOC trial, the follow-up UK Standardization of Breast Radiotherapy Trial A (START A) was initiated in 1999 with a similar design as the RMC/GOC trial except with the 42.9 Gy arm reduced to 41.6 Gy arm $[11,12]$. Eligibility criteria included T1-3a N0-1 M0 invasive breast cancers in patients greater than 18 years of age who underwent BCS or mastectomy with surgical margins $\geq 1 \mathrm{~mm}$. Between 1999 and 2002, 2,236 patients were randomized to one of three arms that were all delivered over five weeks: CF-WBI (50 Gy in 25 fractions) or two HF-WBI arms (41.6 Gy in 13 fractions or 39 Gy in 13 fractions, each delivered every other day). An elective non-randomized boost of $10 \mathrm{~Gy}$ in 5 fractions was given per departmental policy. Twenty-three percent of patients were less than 50 years of age, $15 \%$ underwent mastectomy, $35 \%$ received chemotherapy, $14 \%$ received RNI, and $61 \%$ received a boost. At 10 years, local relapse was $6.7 \%$ in the $50 \mathrm{~Gy}$ arm, $5.6 \%$ in the $41.6 \mathrm{~Gy}$ arm, and $8.1 \%$ in the $39 \mathrm{~Gy}$ arm; neither HF-WBI arm was significantly different from the control CF-WBI arm. Similarly, distant relapse and overall survival did not significantly differ between either of the HF-WBI regimens and CF-WBI regimen. At 10 years, breast induration, edema, and telangiectasias were significantly reduced in the $39 \mathrm{~Gy}$ arm compared to the $50 \mathrm{~Gy}$ arm, while there were no significant differences between the $41.6 \mathrm{~Gy}$ and 50 Gy arm. No differences were noted in breast shrinkage, shoulder stiffness, and arm edema between either HF-WBI arm and CF-WBI. 
The START B trial was initiated simultaneously with START A as a more pragmatic trial with shortened treatment time for the HF-WBI arm $[12,13]$. Eligibility criteria were identical to START A. Between 1999 and 2001, 2,215 patients were randomized to CF- WBI (50 Gy in 25 fractions delivered over five weeks) or HF-WBI ( 40 Gy in 15 fractions delivered over three weeks). An elective non-randomized boost of $10 \mathrm{~Gy}$ in 5 fractions was given per departmental policy. Twenty-one percent of patients were less than 50 years of age, $8 \%$ underwent mastectomy, $22 \%$ received chemotherapy, $7 \%$ received RNI, and $43 \%$ received a boost. At 10 years, local relapse was $5.2 \%$ in the $50 \mathrm{~Gy}$ arm and $3.8 \%$ in the $40 \mathrm{~Gy}$ arm, which were not significantly different. Interestingly, distant relapse (16.0\% vs. $12.3 \%$, $\mathrm{p}=0.014)$ and overall mortality $(19.2 \%$ vs. $15.9 \%, \mathrm{p}=0.042)$ were significantly higher in the CF-WBI arm compared to the HF-WBI arm. However, as these differences were seen early on without a difference in local relapse, it was thought that distant relapse and survival outcomes were not attributable to the treatment regimen. At 10 years, breast shrinkage, telangiectasias, and edema were significantly reduced in the $40 \mathrm{~Gy}$ arm compared to the 50 Gy arm, while there were no significant differences in breast induration, shoulder stiffness, or arm edema.

\section{Recently Closed Randomized Studies Comparing HF-WBI to CF-WBI}

A number of additional randomized trials have been initiated in the past 15 years that have attempted to further define the clinical applicability of HF-WBI as compared to CF-WBI for early-stage breast cancer. These trials have recently closed and major results concerning efficacy are expected in the near future.

In 2004, the UK initiated the FAST trial comparing extremely condensed HF-WBI to CFWBI with a primary endpoint of change in photographic breast appearance [14]. Eligibility criteria included T1-2 N0 M0 invasive breast cancers in patients $\geq 50$ years of age who underwent BCS with microscopic negative margins and tumors less than $3.0 \mathrm{~cm}$ in size. RNI and boost were not allowed. From 2004 to 2007, 915 patients were randomized to one of three dose regimens: CF-WBI (50 Gy in 25 fractions) or one of two HF-WBI arms (30 Gy or $28.5 \mathrm{~Gy}$ in five once-weekly fractions of $6 \mathrm{~Gy}$ or $5.7 \mathrm{~Gy}$, respectively). This trial was unique in using extremely hypofractionated regimens for the experimental arms, which have not been tested in the breast before. Two-year and three-year breast cosmesis was significantly worse for the $30 \mathrm{~Gy}$ arm compared to the $50 \mathrm{~Gy}$ arm $(\mathrm{p}<0.001)$ but there were no significant differences between the $28.5 \mathrm{~Gy}$ and $50 \mathrm{~Gy}$ arms. Tumor control is a secondary endpoint of this trial but has not yet been reported.

In Australia and New Zealand, the Trans-Tasman Radiation Oncology Group (TROG) launched TROG 07.01, the first randomized trial to assess hypofractionation for patients with ductal carcinoma in-situ (DCIS) [15]. Eligibility criteria included patients $\geq 18$ years of age with DCIS who underwent BCS with margins $\geq 1 \mathrm{~mm}$ who are at high risk of local recurrence. High risk was defined as patients less than 50 years of age, or patients greater than 50 years of age with one of the following risk factors: symptomatic presentation, palpable tumor, multifocal disease, microscopic tumor size $\geq 1.5 \mathrm{~cm}$ in maximum dimension, intermediate or high nuclear grade, central necrosis, comedo histology, or radial surgical resection margin less than $10 \mathrm{~mm}$. From 2007 to 2014, 1,608 patients were 
randomized to one of the four trial arms: CF-WBI (50 Gy in 25 fractions) versus HF-WBI (42.5 Gy in 16 fractions) with or without a tumor bed boost. The trial's primary endpoint is time to local recurrence; as of yet results have not been reported.

In the United States (US), the MD Anderson Cancer Center (MDACC) initiated a smaller randomized trial of HF-WBI versus CF-WBI aiming to assess differences in physicianassessed toxicity and patient-reported quality of life $[16,17]$. Eligibility criteria included patients $\geq 40$ years of age with DCIS or T1-2 N0-1a M0 invasive cancer who underwent BCS with negative margins on ink. Between 2011 and 2014, 287 patients were randomized to CF-WBI (50 Gy in 25 fractions plus a boost) or HF-WBI (42.56 Gy in 16 fractions plus a boost). Overall acute grade two ( $47 \%$ vs. $78 \%$, p $<0.001)$ and grade three $(0 \%$ vs. $5 \%$, $\mathrm{p}=0.01$ ) toxicities were lower with HF-WBI compared to CF-WBI, with significantly lower individual components of fatigue, pruritus, breast pain, dermatitis, and hyperpigmentation. There were no differences in acute grade two or grade three toxicity between HF-WBI and CF-WBI in sub-analyses of patients in the upper quartile of body mass index or breast central axis separation. At six months, there were no significant differences in grade two or grade three toxicity and most individual components; however, patients randomized to HFWBI reported less fatigue and less trouble meeting family needs. By two years, patients in both group had improved functional outcomes and significant differences between groups were no longer seen after adjusting for time and baseline functional status.

The Danish Breast Cancer Collaborative Group (DBCG) initiated the randomized HYPO trial with a primary endpoint of grade two or three breast induration at three years [18]. Eligibility criteria included patients greater than 40 years of age with DCIS or T1-2 N0-1(mi) M0 invasive cancer who underwent BCS. From 2009 to 2014, 1,883 patients were randomized to CF-WBI (50 Gy in 25 fractions) or HF-WBI (40 Gy in 16 fractions). Boost was allowed but not randomized. Preliminary results recently published in abstract form demonstrated that overall induration at three years was low (12\%) and did not differ by fractionation regimen, chemotherapy receipt, or use of boost. The only factor that was related to significantly higher induration was large breast size, regardless of HF-WBI or CFWBI regimen [19].

\section{Clinical Implications of HF-WBI}

\subsection{Efficacy of HF-WBI}

Across the four major randomized trials comparing HF-WBI to CF-WBI, a total of 7,095 patients were enrolled, including 4,159 patients on HF-WBI arms, with long-term follow-up. No trial reported any significant differences in 10-year local control between HF-WBI and CF-WBI. No trial reported any significant differences in 10-year distant control or overall survival between HF-WBI and CF-WBI, except as previously noted, in the START B trial with outcomes favoring the HF-WBI arm. These findings were not hypothesized to be attributable to differences in radiation regimens [12]. A Cochrane review published in 2016 demonstrated no significant difference in local recurrence-free survival, relapse-free survival, breast cancer-specific survival, or overall survival between HF-WBI and CF-WBI [20]. Additional meta-analyses have confirmed these findings [21-23]. 


\subsection{Acute Toxicity}

None of the major trials reported on acute toxicity; however, in the MDACC trial, overall acute grade two and three effects were significantly reduced with HF-WBI as compared to CF-WBI, and several patient-reported functional outcomes were improved at six months [16]. Additionally, in a prospective observational study of 2,309 patients, patients who received HF-WBI had significantly lower physician-assessed moist desquamation (6.6\% vs. $28.5 \%, \mathrm{p}<0.001)$ and any grade two or higher dermatitis $(27.4 \%$ vs. $62.6 \%, \mathrm{p}<0.001)$ compared to those who received CF-WBI [24]. Patient-reported metrics demonstrated significantly lower pain, burning/stinging, swelling, and fatigue in those receiving HF-WBI compared to CF-WBI.

\subsection{Late Toxicity}

Across the four major randomized trials, late breast, skin, and subcutaneous tissue toxicity either favored HF-WBI or were similar between HF-WBI and CF-WBI, with the exception of the $42.9 \mathrm{~Gy}$ arm in the RMH/GOC trial that was discontinued for the follow-up START A trial. In the clinically relevant $40 \mathrm{~Gy}$ arm of the START B trial, breast shrinkage, telangiectasias, and edema were improved at 10 years compared to CF-WBI, with other measures of late toxicity showing no difference [12]. No differences in late skin or subcutaneous tissue toxicity were seen in the OCOG trial [10].

Long-term analysis of the START A and B trials additionally showed very low rates of ischemic heart disease $(0.8 \%$ and $1.1 \%$, respectively), symptomatic rib fractures $(0.1 \%$ and $0.3 \%)$, and radiation pneumonitis $(0.1 \%$ and $0.5 \%)$, with no differences between HF-WBI and CF-WBI arms [12]. There was only one reported case of brachial plexopathy amongst all 4,451 patients in the START trials. There were 26 and 17 cardiac deaths in the START A and B trials, respectively, with no significant difference between HF-WBI and CF-WBI. Similarly, long-term update of the OCOG trial reported 21 total cardiac deaths with no significant differences between arms [10].

In all of the major randomized trials, fluoroscopic simulation and two-dimensional planning techniques with physical wedges were used. With modern planning techniques, including computed tomography (CT)-based three-dimensional planning, prone positioning, and deepinspiration breath hold (DIBH) or respiratory gating, the incidence of radiation-related late toxicities should be further minimized whether using HF-WBI or CF-WBI. A prospective trial examined the use of prone versus supine position and found that prone positioning was associated with decreased volume of irradiated lung in all patients and irradiated heart in 85\% of patients with left-sided breast cancer [25]. However, additional studies have found that DIBH in the supine position may offer better cardiac sparing than the free-breathing prone position. The randomized UK HeartSpare study demonstrated that HF-WBI delivered in the supine position with DIBH resulted in lower mean heart and left anterior descending coronary artery doses than in the prone position [26]. With any of these newer techniques, it is clear that normal structures are receiving far less radiation dose than in the past generation of trials. For example, a widely-cited study by Darby and colleagues in 2013 reported a 7.4\% increase in major coronary events per Gy increase in mean heart dose [27], however the average mean heart dose of all 1,144 patients with left-sided cancers included in this 
study was $6.6 \mathrm{~Gy}$ - more than five times the average mean heart dose of $1.2 \mathrm{~Gy}$ reported for patients on the DBCG trial who were treated with HF-WBI and respiratory gating [28].

\subsection{Breast Cosmesis}

In the START B trial, patients randomized to the HF-WBI were less likely to have a change in photographic breast appearance compared to CF-WBI at five years [13]. At 10 years, meta-analysis of all patients on the START trials assessing moderate or marked change breast change favored patients randomized to HF-WBI over CF-WBI [12]. In the OCOG trial, breast cosmesis at 10 years was not significantly different between HF-WBI and CFWBI, rated as good or excellent in about $70 \%$ of patients in both arms [10]. With the development of three-dimensional planning using forward-planned field-in-field techniques or inverse-planned intensity modulated RT (IMRT) to reduce hot spots and dose inhomogeneity, breast cosmesis and other normal tissue effects have further improved [2933].

\section{Clinical Applications of HF-WBI for Early-Stage Breast Cancer}

Based on the available long-term randomized evidence summarized above, the most widely used hypofractionated regimens are $40 \mathrm{~Gy}$ in 15 daily fractions and $42.56 \mathrm{~Gy}$ in 16 daily fractions, with indications for a tumor bed boost (10 Gy in four or five daily fractions) that are identical to the conventional setting. Thus, standard HF-WBI courses last three to four weeks depending on use of a boost. There are few scenarios in which women with earlystage breast cancer undergoing BCT are not eligible for HF-WBI; below we discuss some common clinical situations in which there may still be apprehension to adopt HF-WBI.

\subsection{HF-WBI in Younger Patients}

Across the four major randomized trials comparing HF-WBI to CF-WBI, 1,694 patients less than 50 years of age were enrolled, representing $24 \%$ of total enrollment. Sub-group analysis of the OCOG trial demonstrated that there was no significant difference in 10-year local recurrence for patients less than 50 years of age between HF-WBI and CF-WBI (hazard ratio $(\mathrm{HR})=0.77 ; 95 \%$ confidence interval $(\mathrm{CI})$ : $0.35-1.70)$. Analysis of cosmetic outcomes found that age $<50$ years was a significant predictor of having a good or excellent cosmetic outcome regardless of treatment regimen, and there was no interaction between age and treatment regimen on cosmetic outcome [10]. Similarly, sub-group analysis of all patients on the RMH/GOC, START A, and START B trials showed that there was no difference in longterm locoregional control for patients less than $40(\mathrm{HR}=0.79 ; 95 \%$ CI: $0.47-1.34)$ or between 40 and 49 years of age (HR=0.88; 95\% CI: 0.60-1.28) by treatment arm. There was also no difference in cosmetic outcome between treatment arms for these two age groups [12]. These results demonstrate that HF-WBI can be offered to patients of any age, with the caveat that these trials have only reported ten-year follow-up data compared to 25 or more years of follow-up for CF-WBI trials. However, given the available evidence and the known radiobiological parameters of hypofractionation, it is unlikely that differences favoring CFWBI will emerge with longer follow-up. 


\subsection{HF-WBI for High-Grade or Estrogen Receptor-Negative Tumors}

The OCOG trial was notable for its sub-group analysis showing that for high-grade tumors, 10-year cumulative local recurrence was $15.6 \%$ in the HF-WBI arm compared to $4.7 \%$ in the CF-WBI arm (HR=3.08, 95\% CI: 1.22-7.76) [10]. There was a significant interaction between treatment arm and tumor grade $(\mathrm{p}=0.01)$. However, the original trial used the antiquated Scharff Bloom Richardson grading system, and after central pathologic review of tumor blocks from 989 patients using the more modern Nottingham grading system, tumor grade was not predictive of response to hypofractionation [34]. Further, molecular subtype (luminal A, luminal B, HER2 enriched, basal-like, or unclassified) was not found to be predictive of response to hypofractionation. The meta-analysis of START trials did not show high-grade tumors to have any significant difference in local recurrence between HF-WBI and CF-WBI (HR=0.86; 95\% CI: 0.59-1.25) [12]. These results together suggest that hypofractionation is safe to use in patients with high-grade and/or estrogen receptor-negative tumors.

\subsection{HF-WBI with Tumor Bed Boost}

Across the RMH/GOC, START A, and START B trials, 3,078 patients (53\%) received a boost of either $14 \mathrm{~Gy}$ in 10 fractions in the RMH/GOC trial or $10 \mathrm{~Gy}$ in 5 fractions in the START trials. In subgroup analysis of those who received a boost, there was no difference in local recurrence at 10 years (HR=0.99, 95\% CI: 0.76-1.29) and there was less moderate or marked breast changes in patients randomized to HF-WBI compared to CF-WBI (HR $=0.86$, 95\% CI: 0.76-0.96) [12]. Additionally, the DBCG HYPO trial did not show that boost was related to breast induration at three years irrespective of treatment regimen [19]. These results demonstrate that a boost is safe to use with HF-WBI and should be considered as part of treatment with the same criteria as those for CF-WBI.

\subsection{HF-WBI in Patients with Larger Breasts}

OCOG eligibility criteria limited inclusion to patients with breast maximum central axis separation $\leq 25 \mathrm{~cm}$ [9]. This was done in the era of two-dimensional planning to allow for acceptable dose homogeneity, which is correlated with acute and late toxicity $[32,33]$. The UK trials did not place a limit on breast size [11,13]. With modern CT-based threedimensional planning techniques, as described above, there is less concern that dose homogeneity goals will not be achievable in patients with larger breasts, or that dose inhomogeneity will create more adverse effects with HF-WBI over CF-WBI. Meta-analysis of the START trials did not show any difference in cosmetic outcome for patients with larger breasts between HF-WBI and CF-WBI [12]. Additionally, the MDACC trial, which used three-dimensional planning, did not demonstrate higher acute side effects in patients in the upper quartile of breast central axis separation or body mass index receiving HF-WBI as compared to CF-WBI [16], demonstrating the safety of HF-WBI in patients with large breasts.

\subsection{HF-WBI with Systemic Treatment}

Across the four major randomized trials, 1,616 patients (23\%) received chemotherapy, although almost no patients received taxane-based regimens, which is now standard practice 
in breast therapy. The OCOG trial did not show any difference in long-term local recurrence between treatments arms in those who received chemotherapy (HR=1.06; 95\% CI:

0.58-1.97) and did not show any interaction between treatment and receipt of chemotherapy on cosmetic outcome [10]. Likewise, the meta-analysis of RMH/GOC and START trials did not show any difference in locoregional recurrence in those who received chemotherapy between HF-WBI and CF-WBI (HR=0.81; 95\% CI: 0.57-1.14). In those who received chemotherapy, cosmetic outcomes were not different between arms, while in those who received tamoxifen there was better cosmesis in patients randomized to HF-WBI $(\mathrm{HR}=0.84$; 95\% CI: 0.76-0.93) [12]. These results demonstrate that systemic treatment with chemotherapy or hormonal therapy is safe in conjunction with HF-WBI.

\subsection{HF-WBI for DCIS}

Similar to invasive breast cancer, multiple large randomized trials have demonstrated the benefit of CF-WBI in decreasing locoregional recurrence after BCS for DCIS [35-38]. An EBCTCG meta-analysis of 3,729 women with DCIS in 4 randomized trials found that adjuvant RT after BCS reduced 10-year risk of recurrence from $28.1 \%$ to $12.9 \%$ with no significant effect on breast cancer-specific or all-cause mortality [39].

However, to date there is no randomized data comparing HF-WBI to CF-WBI for DCIS. None of the major trials included patients with pure DCIS, although the OCOG trial allowed a DCIS component to invasive disease [9]. The DBCG trial did allow DCIS; however, only a small minority of patients enrolled had DCIS (13\%), and tumor control results have not yet been reported [19]. A number of retrospective reports have found excellent local control outcomes with HF-WBI for DCIS [40,41] and equivalence between HF-WBI and CF-WBI [42-44]. A meta-analysis of 2,534 patients with DCIS on four studies found no difference in local control between HF-WBI and CF-WBI (odds ratio $(\mathrm{OR})=0.78$; 95\% CI: 0.58-1.03) however with a low level of evidence [45]. Based on these studies and extrapolation from the major randomized studies of invasive disease, there is widespread empirical use of HF-WBI for DCIS; however, the recently closed TROG 07.01 trial will provide a definitive evidentiary basis as to the applicability of hypofractionated treatment for DCIS [15].

\section{Consensus Guidelines}

The American Society for Radiation Oncology (ASTRO) published consensus guidelines in 2011 endorsing HF-WBI for patients 50 years or older at diagnosis, with T1-2 N0 invasive cancer treated with BCS, who did not receive chemotherapy, and in whom central axis dose homogeneity within $\pm 7 \%$ of the prescription dose could be achieved [46]. For the remainder of patients not meeting all of these criteria, the guidelines permitted, but did not specifically endorse or prohibit, HF-WBI. However, these guidelines were published before long-term data from the START trials were available [12] and subsequently most deemed the criteria too conservative.

ASTRO published updated consensus guidelines in 2018, endorsing HF-WBI as a new standard of care in most women with early stage breast cancer who do not require regional nodal irradiation [47]. Specifically, the guidelines state that for women with invasive breast cancer receiving whole breast irradiation with or without inclusion of the low axilla, the 
preferred dose-fractionation scheme is HF-WBI to a dose of 40 Gy in 15 fractions or 42.5 Gy in 16 fractions. The guidelines also state that hypofractionation may be used as an alternative to conventional fractionation for patients with DCIS. Further, the guidelines state that the decision to offer hypofractionation should be independent of grade, laterality, administration of systemic therapy, age, or breast size provided acceptable dose homogeneity can be achieved. The authors note, however, that the guidelines are intended as a "tool to promote appropriately individualized, shared decision-making between physicians and patients, and should not be construed as strict or superseding the appropriately informed and considered judgments of individual physicians and patients."

\section{Practical Implementation of HF-WBI}

\subsection{Current Adoption}

Despite strong level-one evidence supporting the use of HF-WBI, its uptake has been slow in the US. In a study of administrative claims data covering 15,643 women, utilization was assessed on the basis of 2011 ASTRO consensus guidelines. Despite increasing utilization from 2008 to 2013, HF-WBI was only used in 34.5\% of hypofractionation-endorsed patients and $21.2 \%$ of hypofractionation-permitted patients in 2013 [48]. A study of the National Cancer Database found similar findings with HF-WBI usage among patients with invasive cancer increasing from $0.7 \%$ to $15.6 \%$ from 2004 to 2013 [49]. While it is encouraging to see increased adoption, the most recent estimates available still suggest a third or less of eligible patients are receiving HF-WBI. In contrast, under the UK's national health system, HF-WBI is used almost universally, and in Canada, HF-WBI is used in about $71 \%$ of BCT cases [50,51]. Other European countries have shown far lower uptake rates of HF-WBI compared to the UK [51,52]. Many reasons for poor uptake in the US have been offered, including ASTRO's conservative 2011 recommendations, a high proportion of obese patients, hesitation to change practice with only ten-year follow-up data, and finally the US fee-for-service reimbursement structure that incentivizes the use of more fractions, rather than less. The goal of increasing utilization of HF-WBI has led ASTRO to revisit its consensus guidelines and recommend HF-WBI as a preferred fractionation scheme [47].

\subsection{Costs and Resource Utilization}

HF-WBI can reduce healthcare costs by about a third compared to CF-WBI [48,53]. In one study, HF-WBI resulted in direct US Medicare costs of $\$ 7,826$ compared to $\$ 11,725$ for CFWBI, creating a $33 \%$ reduction in costs [54]. Indirect cost savings additionally benefit the patient in the form of transportation costs, opportunity costs, and lost productivity from extended treatment schedules. A recent cost-effectiveness analysis found HF-WBI to have reduced overall costs and increased patient quality-adjusted life years compared to CF-WBI [55]. Further, in many countries, there exist long waiting times for RT [56,57] and a full transition from CF-WBI to HF-WBI can increase system throughput, reduce waiting times, and increase the number of patients that are treatable in a system of fixed resources $[58,59]$.

\subsection{Access to Care}

About 35\% of patients who are eligible for BCS undergo mastectomy [60] and only about $65-80 \%$ of patients who undergo BCS or mastectomy receive adjuvant RT [61,62]. It has 
been theorized that the long treatment course of CF-WBI may be an underlying reason that patients choose mastectomy over BCT or choose to forgo RT after surgery. An analysis of the Surveillance, Epidemiology, and End Results database found that living 15 miles or more away from the nearest radiotherapy facility was associated with a decreased likelihood of receiving $\mathrm{BCS}(\mathrm{OR}=0.52 ; 95 \% \mathrm{CI}=0.46-0.58)$ and living 40 miles or more away was associated with a decreased likelihood of receiving RT after BCS (OR $=0.55 ; 95 \%$ $\mathrm{CI}=0.37-0.82$ ) [63]. If HF-WBI is more feasible for patients, there exists potential for HFWBI adoption to lead more patients to choose BCT rather than mastectomy and to increase access to or compliance with adjuvant RT schedules after surgery. Indeed, a recent National Cancer Database study demonstrated that increased adoption of HF-WBI in patients with DCIS who lived 50 or more miles away from the nearest hospital increased overall use of RT [49].

\section{Hypofractionation for Locally Advanced Breast Cancer}

Although the standard of care for early-stage breast cancer is BCT, many women with locally advanced breast cancer still require mastectomy. Post-mastectomy RT (PMRT) was historically limited to patients with four or more positive nodes, although recent data has shown improved locoregional control and survival in post-mastectomy patients with any number of positive nodes [64-66]. As a result, use of PMRT has been increasing in the last decade, and most patients who undergo PMRT will require regional nodal irradiation (RNI) $[67,68]$. RNI generally includes radiation to the axillary, supraclavicular, and/or internal mammary nodes, putting additional tissues such as the brachial plexus, heart, and lungs at risk of radiation-related toxicity.

Early attempts at hypofractionated PMRT were made in the 1960s and 1970s, with an unacceptable number of patients experiencing shoulder dysfunction, arm edema, lung fibrosis, brachial plexopathy, and paralysis [69-71]. However, these studies increased the daily dose of radiation without appropriately modifying total dose and used archaic beam planning that caused hot spots in the supraclavicular area, resulting in extremely high radiobiological doses to normal tissues including the brachial plexus. Although radiobiological concepts are better understood now and planning techniques have become much more sophisticated, these studies have highlighted the reluctance to hypofractionate when treating the supraclavicular area, whether after BCS or mastectomy. An additional issue that needs to be resolved in the post-mastectomy setting is whether hypofractionation exacerbates breast reconstruction complications, which is already seen with conventionallyfractionated PMRT [72,73].

The vast majority of patients enrolled on the major randomized trials had early-stage T1-2 N0 tumors and underwent BCS, with only a minority of all patients receiving RNI (11\%). Long-term follow-up of the START trials did not show any increased rates of shoulder stiffness or arm edema in those receiving RNI with HF-WBI versus CF-WBI [12]. Further, there was only one case of brachial plexopathy amongst all 4,451 patients, demonstrating that modern techniques for hypofractionated RNI are much safer than prior attempts. 
A recent single-arm, prospective phase II study tested hypofractionated PMRT including RNI (36.63 Gy in 11 fractions with an optional scar boost of 12.32 Gy in 4 fractions) in 69 patients with Stage II-IIIC breast cancer [74]. Three-year local-and distant-recurrence free survival were $89.2 \%$ (95\% CI: 0.749-0.956) and 90.3\% (95\% CI: 0.797-0.956), respectively. There were no grade three toxicities, no cases of brachial plexopathy, three cases of grade 2 lymphedema, and the reconstruction complication rate was similar to rates seen with CFWBI. Another Phase II trial studied hypofractionated RNI delivered in the prone position after BCS or mastectomy in 69 patients with Stage IB-IIIA breast cancer [75]. The dose regimen consisted of $40.5 \mathrm{~Gy}$ in 15 fractions with a concurrent daily boost of $0.5 \mathrm{~Gy}$ for a total of $48 \mathrm{~Gy}$ to the tumor bed. Three-year breast cancer-specific survival was $95.6 \%$ with no locoregional recurrences after a median of 35 months. This trial also demonstrated favorable late effects, with only a single late grade 3 toxicity, one grade 2 lymphedema, and no cases of brachial plexopathy.

A randomized phase III trial (Alliance A221505) was recently opened comparing hypofractionated PMRT (42.56 Gy in 16 fractions) to conventionally-fractionated PMRT (50 Gy in 25 fractions) [76]. Eligibility criteria include patients $\geq 18$ years of age with Stage IIIIIA (all node-positive) invasive breast cancer who undergo mastectomy with immediate or delayed reconstruction. RNI of the same dose regimen is mandatory while neoadjuvant or adjuvant chemotherapy are allowed and scar boost is not allowed. The target enrollment is 880 patients with a primary endpoint of reconstruction complication rate and secondary endpoints of tumor control and toxicity outcomes.

Investigators from the Chinese Academy of Medical Sciences also recently completed a randomized trial comparing hypofractionated PMRT ( 43.5 Gy in 15 fractions) to conventionally-fractionated PMRT (50 Gy in 25 fractions) with RNI and no scar boost. From 2008 to 2016, patients with primarily stage III disease were enrolled and randomized. Initial results were presented at the 2017 ASTRO annual meeting [77]. There were no significant differences in five-year locoregional recurrence ( $8.4 \%$ vs. $6.0 \% ; \mathrm{p}=0.396$ ), disease-free survival $(75.1 \%$ vs. $74.6 \%$; $\mathrm{p}=0.841)$, or overall survival $(84.9 \%$ vs. $87.1 \%$; $\mathrm{p}=0.562$ ). Similarly, incidence of radiation pneumonitis, lymphedema, shoulder dysfunction, and ischemic heart disease were similar between groups, while incidence of grade three acute skin toxicity was lower in the hypofractionated group. There were no cases of brachial plexopathy. One drawback of this trial is that breast reconstruction was not allowed. More than a third of patients undergoing mastectomy in the US in 2011 opted for breast reconstruction, with an increasing trend [60], making it difficult to extrapolate these results to the reconstructed population.

The results from these trials are encouraging, however follow-up remains short for the majority of these patients, with the exception of the few patients on the START trials. Broad adoption of hypofractionation with RNI in the locally advanced or post-mastectomy setting awaits longer follow-up from these and other ongoing clinical trials. 


\section{Conclusion}

Breast conservation therapy, consisting of breast conservation surgery followed by adjuvant RT, has been the standard of care for early-stage breast cancer for decades. CF-WBI over a course of five to seven weeks was historically used to spare normal tissues, but numerous randomized trials have demonstrated that shorter courses of HF-WBI are an appropriate replacement for CF-WBI in most patients who do not require regional nodal irradiation. The most commonly-used HF-WBI regimens of 40 Gy in 15 fractions and 42.56 Gy in 16 fractions over three weeks have been demonstrated to result in equivalent tumor control outcomes, improved or similar acute and late toxicity, and improved or similar long-term breast cosmesis compared to CF-WBI regimens of $50 \mathrm{~Gy}$ in 25 fractions. When including a tumor bed boost of $10 \mathrm{~Gy}$ in four to five fractions, these regimens are generally completed in about four weeks. The use of hypofractionated regimens in the locally-advanced setting is evolving with ongoing randomized trials evaluating efficacy and safety compared to conventional regimens. Overall, hypofractionation in breast cancer therapy represents highquality and high-value care that is not only more convenient for patients, but also more costeffective and resourceful for the healthcare system as a whole.

\section{Expert Commentary}

The OCOG and START trials are considered to be practice-changing in the field of breast radiation oncology. Whereas historically it was standard to deliver a course of whole breast radiotherapy over five to seven weeks, with long-term data from these trials it has become well-accepted that shorter hypofractionated courses of three to four weeks are equivalent in tumor control and may even improve toxicity and breast cosmesis. Long-term results demonstrate that there are very few disadvantages of HF-WBI, and as such, have led ASTRO to revise its consensus guidelines to recommend HF-WBI as standard practice in most women with early-stage breast cancers.

It would not be surprising if the Alliance trial changes the field of post-mastectomy radiation treatment in the way the OCOG and START trials changed the field of post-BCS RT. The use of hypofractionation with regional nodal irradiation is not yet the standard of care, however early data indicate that it is safe and unlikely to result in the brachial plexus toxicities that were seen decades ago with antiquated techniques. Long-term data from the Alliance trial will be needed to draw definitive conclusions and bring hypofractionated PMRT into mainstream clinical practice.

The advent of HF-WBI brings many opportunities in creating safer, more effective, and more convenient treatment regimens that increase patients' access to care and ability to undergo breast conservation. Additional opportunities are created for the healthcare system to reduce costs and utilize resources more efficiently, which will be ever more important as the number of patients with breast cancer is expected to continue to increase.

ASTRO recently chose shared decision-making of HF-WBI as one of five goals in its Choosing Wisely campaign, highlighting its importance to patients and providers alike. 
Hypofractionation in breast cancer represents innovative, high-value, high-quality care at the forefront of cancer treatment and will continue to evolve in the coming years.

\section{Five-year view}

HF-WBI may see a turning point over the next five years in which its uptake is greatly expanded across the US and other countries where it is not yet widely utilized as evidence supporting its use continues to build. In the US, stronger ASTRO consensus recommendations combined with upcoming expected payment reforms will promote greater use of HF-WBI.

There is clear interest in creating even shorter and more condensed HF-WBI regimens. The UK FAST trial tested extremely hypofractionated regimens against conventional fractionation and found that five once-weekly fractions of $5.7 \mathrm{~Gy}$ did not result in inferior two- or three-year cosmetic outcome compared to CF-WBI [14]; however, tumor control outcomes have not yet been reported and will likely be available in the next several years. The follow-up FAST-Forward trial is using a 40 Gy in 15 fraction HF-WBI as a new standard control arm (given the high level of evidence from the first generation of trials) and testing extremely hypofractionated regimens of $5.4 \mathrm{~Gy}$ or $5.2 \mathrm{~Gy}$ given in five daily fractions to the whole breast, completing treatment in one week. This trial is also enrolling patients who require PMRT and/or RNI and will thus have great implications for adjuvant breast RT in all patients [78].

In the US, a smaller phase II trial demonstrated the efficacy and safety of completing a three-week course of HF-WBI inclusive of a sequential boost [79], similar to the PMRT trial that was completed in three weeks [74]. Three-week courses of HF-WBI have also been accomplished using a concurrent boost, as in the aforementioned hypofractionated RNI trial which used a hybrid IMRT approach [75]. A randomized trial using a HF-WBI dose regimen of $40.5 \mathrm{~Gy}$ in 15 fractions compared daily $0.5 \mathrm{~Gy}$ boost (total $48 \mathrm{~Gy}$ to the tumor bed) to weekly 2 Gy boost (total 46.5 Gy) using IMRT, and at a median of 45 months found no difference in efficacy, acute or late toxicity, or breast cosmesis [80]. The Radiation Therapy Oncology Group (RTOG) is conducting the RTOG 1005 randomized Phase III trial which is testing HF-WBI of $40 \mathrm{~Gy}$ in 15 fractions with a simultaneous integrated boost to $48 \mathrm{~Gy}$, using optional IMRT, compared to standard CF-WBI or HF-WBI with sequential boost [81]. Long-term results of these trials may lead to concurrent boost becoming an accepted standard approach with HF-WBI.

There are a number of other randomized studies for which results are pending, most notably the TROG 07.01 study which will help to define the acceptability of HF-WBI for patients with DCIS in a randomized setting [15].

As long-term results from the first-generation major randomized have demonstrated the overall superiority of HF-WBI compared to CF-WBI, the next generation of trials will take HF-WBI arms from the OCOG and START trials as standard and compare newer techniques including highly condensed schedules, integrated boosts, IMRT, and other innovations within a hypofractionated framework. 


\section{Acknowledgments}

Funding

This paper was supported in part by the National Cancer Institute's Core Center support grant (P30CA072720) and the Breast Cancer Research Foundation.

\section{References}

Reference annotations

* Of interest

** Of considerable interest

1. Ferlay J, Soerjomataram I, Dikshit R, et al. Cancer incidence and mortality worldwide: sources, methods and major patterns in GLOBOCAN 2012. Int J cancer. 2015;136(5):E359-386. [PubMed: 25220842]

2. Fisher B, Anderson S, Bryant J, et al. Twenty-Year Follow-up of a Randomized Trial Comparing Total Mastectomy, Lumpectomy, and Lumpectomy plus Irradiation for the Treatment of Invasive Breast Cancer. N Engl J Med. 2002;347(16):1233-1241. [PubMed: 12393820]

3. Veronesi U, Cascinelli N, Mariani L, et al. Twenty-Year Follow-up of a Randomized Study Comparing Breast-Conserving Surgery with Radical Mastectomy for Early Breast Cancer. N Engl J Med. 2002;347(16):1227-1232. [PubMed: 12393819]

4. Clark RM, Whelan T, Levine M, et al. Randomized clinical trial of breast irradiation following lumpectomy and axillary dissection for node-negative breast cancer: an update. Ontario Clinical Oncology Group. J Natl Cancer Inst. 1996;88(22):1659-1664. [PubMed: 8931610]

5. Liljegren G, Holmberg L, Bergh J, et al. 10-Year Results After Sector Resection With or Without Postoperative Radiotherapy for Stage I Breast Cancer: A Randomized Trial. J Clin Oncol. 1999;17(8):2326-2333. [PubMed: 10561294]

6. Early Breast Cancer Trialists' Collaborative Group (EBCTCG). Effect of radiotherapy after breastconserving surgery on 10-year recurrence and 15-year breast cancer death: meta-analysis of individual patient data for 10,801 women in 17 randomised trials. Lancet. 2011;378(9804):17071716. [PubMed: 22019144]

7. Yarnold J, Ashton A, Bliss J, et al. Fractionation sensitivity and dose response of late adverse effects in the breast after radiotherapy for early breast cancer: long-term results of a randomised trial. Radiother Oncol. 2005;75(1):9-17. [PubMed: 15878095]

8. Owen JR, Ashton A, Bliss JM, et al. Effect of radiotherapy fraction size on tumour control in patients with early-stage breast cancer after local tumour excision: long-term results of a randomised trial. Lancet Oncol. 2006;7(6):467-471. [PubMed: 16750496]

9. Whelan T, MacKenzie R, Julian J, et al. Randomized trial of breast irradiation schedules after lumpectomy for women with lymph node-negative breast cancer. J Natl Cancer Inst. 2002;94(15): 1143-1150. [PubMed: 12165639]

10. Whelan TJ, Pignol JP, Levine MN, et al. Long-Term Results of Hypofractionated Radiation Therapy for Breast Cancer. N Engl J Med. 2010;362(6):513-520. [PubMed: 20147717] ** Longterm outcomes of the OCOG trial comparing hypofractionated to conventional whole breast irradiation.

11. START Trialists' Group. The UK Standardisation of Breast Radiotherapy (START) Trial A of radiotherapy hypofractionation for treatment of early breast cancer: a randomised trial. Lancet Oncol. 2008;9(4):331-341. [PubMed: 18356109]

12. Haviland JS, Owen JR, Dewar JA, et al. The UK Standardisation of Breast Radiotherapy (START) trials of radiotherapy hypofractionation for treatment of early breast cancer: 10-year follow-up results of two randomised controlled trials. Lancet Oncol. 2013;14(11):1086-1094. [PubMed: 24055415 ]** Long-term outcomes and combined analysis of the START A and B trials comparing hypofractionated to conventional whole breast irradiation. 
13. START Trialists' Group. The UK Standardisation of Breast Radiotherapy (START) Trial B of radiotherapy hypofractionation for treatment of early breast cancer: a randomised trial. Lancet. 2008;371(9618):1098-1107. [PubMed: 18355913]

14. FAST Trialists group. First results of the randomised UK FAST Trial of radiotherapy hypofractionation for treatment of early breast cancer (CRUKE/04/015). Radiother Oncol. 2011;100(1):93-100. [PubMed: 21752481]

15. Radiation Doses and Fractionation Schedules in Non-low Risk Ductal Carcinoma In Situ (DCIS) of the Breast. National Institute of Health Accessed 2018 Feb 26 Available from: https:// clinicaltrials.gov/ct2/show/NCT00470236

16. Shaitelman SF, Schlembach PJ, Arzu I, et al. Acute and Short-term Toxic Effects of Conventionally Fractionated vs Hypofractionated Whole-Breast Irradiation. JAMA Oncol. 2015;1(7):931-941. [PubMed: 26247543] * Acute toxicity of hypofractionated compared to conventional whole breast irradiation examined in a randomized setting.

17. Swanick CW, Lei X, Shaitelman SF, et al. Longitudinal analysis of patient-reported outcomes and cosmesis in a randomized trial of conventionally fractionated versus hypofractionated whole-breast irradiation. Cancer. 2016;122(18):2886-2894. [PubMed: 27305037]

18. Hypofractionated Versus Standard Fractionated Whole Breast Irradiation to Node-negative Breast Cancer Patients. National Institute of Health Accessed 2018 Feb 27 Available from: https:// clinicaltrials.gov/show/NCT00909818

19. Offersen BV, Nielsen HM, Jacobsen EH, et al. Hypo- vs normofractionated radiation therapy of early stage breast cancer in the randomized DBCG HYPO trial. Eur J Cancer. 2017;72:S9-10.

20. Hickey BE, James ML, Lehman M, et al. Hypofractionated radiation therapy for early breast cancer. Cochrane Database Syst Rev. 2016;7:CD003860. [PubMed: 27425588]

21. Valle LF, Agarwal S, Bickel KE, et al. Hypofractionated whole breast radiotherapy in breast conservation for early-stage breast cancer: a systematic review and meta-analysis of randomized trials. Breast Cancer Res Treat. 2017;162(3):409-417. [PubMed: 28160158]

22. Budach W, Bölke E, Matuschek C. Hypofractionated Radiotherapy as Adjuvant Treatment in Early Breast Cancer. A Review and Meta-Analysis of Randomized Controlled Trials. Breast Care. 2015;10(4):240-245. [PubMed: 26600759]

23. Zhou ZR, Mei X, Chen XX, et al. Systematic review and meta-analysis comparing hypofractionated with conventional fraction radiotherapy in treatment of early breast cancer. Surg Oncol. 2015;24(3):200-211. [PubMed: 26116397]

24. Jagsi R, Griffith KA, Boike TP, et al. Differences in the Acute Toxic Effects of Breast Radiotherapy by Fractionation Schedule: Comparative Analysis of Physician-Assessed and Patient-Reported Outcomes in a Large Multicenter Cohort. JAMA Oncol. 2015;1(7):918-930. [PubMed: 26247417]

25. Formenti SC, DeWyngaert JK, Jozsef G, et al. Prone vs Supine Positioning for Breast Cancer Radiotherapy. JAMA. 2012;308(9):861-863. [PubMed: 22948692]

26. Bartlett FR, Colgan RM, Donovan EM, et al. The UK HeartSpare Study (Stage IB): randomised comparison of a voluntary breath-hold technique and prone radiotherapy after breast conserving surgery. Radiother Oncol. 2015;114(1):66-72. [PubMed: 25739317]

27. Darby SC, Ewertz M, McGale P, et al. Risk of ischemic heart disease in women after radiotherapy for breast cancer. N Engl J Med. 2013;368(11):987-998. [PubMed: 23484825]

28. Berg M, Lorenzen EL, Jensen I, et al. The potential benefits from respiratory gating for breast cancer patients regarding target coverage and dose to organs at risk when applying strict dose limits to the heart: results from the DBCG HYPO trial. Acta Oncol. 2018;57(1):113-119. [PubMed: 29205080]

29. Keller LMM, Sopka DM, Li T, et al. Five-year results of whole breast intensity modulated radiation therapy for the treatment of early stage breast cancer: the Fox Chase Cancer Center experience. Int J Radiat Oncol Biol Phys. 2012;84(4):881-887. [PubMed: 22909414]

30. Freedman GM, Li T, Nicolaou N, et al. Breast intensity-modulated radiation therapy reduces time spent with acute dermatitis for women of all breast sizes during radiation. Int J Radiat Oncol Biol Phys. 2009;74(3):689-694. [PubMed: 19362779] 
31. Harsolia A, Kestin L, Grills I, et al. Intensity-modulated radiotherapy results in significant decrease in clinical toxicities compared with conventional wedge-based breast radiotherapy. Int J Radiat Oncol Biol Phys. 2007;68(5):1375-1380. [PubMed: 17544598]

32. Pignol JP, Olivotto I, Rakovitch E, et al. A multicenter randomized trial of breast intensitymodulated radiation therapy to reduce acute radiation dermatitis. J Clin Oncol. 2008;26(13):20852092. [PubMed: 18285602]

33. Donovan E, Bleakley N, Denholm E, et al. Randomised trial of standard 2D radiotherapy (RT) versus intensity modulated radiotherapy (IMRT) in patients prescribed breast radiotherapy. Radiother Oncol. 2007;82(3):254-264. [PubMed: 17224195]

34. Bane AL, Whelan TJ, Pond GR, et al. Tumor factors predictive of response to hypofractionated radiotherapy in a randomized trial following breast conserving therapy. Ann Oncol Off J Eur Soc Med Oncol. 2014;25(5):992-998.

35. Wapnir IL, Dignam JJ, Fisher B, et al. Long-term outcomes of invasive ipsilateral breast tumor recurrences after lumpectomy in NSABP B-17 and B-24 randomized clinical trials for DCIS. J Natl Cancer Inst. 2011;103(6):478-488. [PubMed: 21398619]

36. Holmberg L, Garmo H, Granstrand B, et al. Absolute risk reductions for local recurrence after postoperative radiotherapy after sector resection for ductal carcinoma in situ of the breast. J Clin Oncol. 2008;26(8):1247-1252. [PubMed: 18250350]

37. Bijker N, Meijnen P, Peterse JL, et al. Breast-Conserving Treatment With or Without Radiotherapy in Ductal Carcinoma-In-Situ: Ten-Year Results of European Organisation for Research and Treatment of Cancer Randomized Phase III Trial 10853 - A Study by the EORTC Breast Cancer Cooperative Group and EORTC Radiotherapy Group. J Clin Oncol. 2006;24(21):3381-3387. [PubMed: 16801628]

38. Cuzick J, Sestak I, Pinder SE, et al. Effect of tamoxifen and radiotherapy in women with locally excised ductal carcinoma in situ: long-term results from the UK/ANZ DCIS trial. Lancet Oncol. 2011;12(1):21-29. [PubMed: 21145284]

39. Early Breast Cancer Trialists' Collaborative Group (EBCTCG). Overview of the Randomized Trials of Radiotherapy in Ductal Carcinoma In Situ of the Breast. JNCI Monogr. 2010;2010(41): 162-177.

40. Hathout L, Hijal T, Théberge V, et al. Hypofractionated Radiation Therapy for Breast Ductal Carcinoma In Situ. Int J Radiat Oncol. 2013;87(5):1058-1063.

41. Ciervide R, Dhage S, Guth A, et al. Five year outcome of 145 patients with ductal carcinoma in situ (DCIS) after accelerated breast radiotherapy. Int J Radiat Oncol Biol Phys. 2012;83(2):e159_ 64. [PubMed: 22579378]

42. Wai ES, Lesperance ML, Alexander CS, et al. Effect of radiotherapy boost and hypofractionation on outcomes in ductal carcinoma in situ. Cancer. 2011;117(1):54-62. [PubMed: 20803608]

43. Lalani N, Paszat L, Sutradhar R, et al. Long-term outcomes of hypofractionation versus conventional radiation therapy after breast-conserving surgery for ductal carcinoma in situ of the breast. Int J Radiat Oncol Biol Phys. 2014;90(5):1017-1024. [PubMed: 25220719]

44. Williamson D, Dinniwell R, Fung S, et al. Local control with conventional and hypofractionated adjuvant radiotherapy after breast-conserving surgery for ductal carcinoma in-situ. Radiother Oncol. 2010;95(3):317-320. [PubMed: 20400190]

45. Nilsson C, Valachis A. The role of boost and hypofractionation as adjuvant radiotherapy in patients with DCIS: a meta-analysis of observational studies. Radiother Oncol. 2015;114(1):50-55. [PubMed: 25596912]

46. Smith BD, Bentzen SM, Correa CR, et al. Fractionation for whole breast irradiation: an American Society for Radiation Oncology (ASTRO) evidence-based guideline. Int J Radiat Oncol Biol Phys. 2011;81(1):59-68. [PubMed: 20638191]

47. Smith BD, Bellon JR, Blitzblau R, et al. Radiation therapy for the whole breast: Executive summary of an American Society for Radiation Oncology (ASTRO) evidence-based guideline. Pract Radiat Oncol. 2018;8(3):145-152. [PubMed: 29545124] ** Recently revised consensus guidelines covering the use of hypofractionated whole breast irradiation in women with early stage breast cancer who do not require regional nodal irradiation. 
48. Bekelman JE, Sylwestrzak G, Barron J, et al. Uptake and Costs of hypofractionated vs conventional whole breast irradiation after breast conserving surgery in the United States, 20082013. JAMA - J Am Med Assoc. 2014;312(23):2542-2550.

49. Hasan Y, Waller J, Yao K, et al. Utilization trend and regimens of hypofractionated whole breast radiation therapy in the United States. Breast Cancer Res Treat. 2017;162(2):317-328. [PubMed: 28120272]

50. Ashworth A, Kong W, Whelan T, et al. A Population-Based Study of the Fractionation of Postlumpectomy Breast Radiation Therapy. Int J Radiat Oncol. 2013;86(1):51-57.

51. van der Laan HP, Hurkmans CW, Kuten A, et al. Current technological clinical practice in breast radiotherapy; results of a survey in EORTC-Radiation Oncology Group affiliated institutions. Radiother Oncol. 2010;94(3):280-285. [PubMed: 20116120]

52. Prades J, Algara M, Espinàs JA, et al. Understanding variations in the use of hypofractionated radiotherapy and its specific indications for breast cancer: A mixed-methods study. Radiother Oncol. 2017;123(1):22-28. [PubMed: 28236538]

53. Lievens Y Hypofractionated breast radiotherapy: Financial and economic consequences. The Breast. 2010;19(3):192-197. [PubMed: 20511069]

54. Lanni T, Keisch M, Shah C, et al. A Cost Comparison Analysis of Adjuvant Radiation Therapy Techniques after Breast-Conserving Surgery. Breast J. 2013;19(2):162-167. [PubMed: 23294184]

55. Deshmukh AA, Shirvani SM, Lal L, et al. Cost-effectiveness Analysis Comparing Conventional, Hypofractionated, and Intraoperative Radiotherapy for Early-Stage Breast Cancer. J Natl Cancer Inst. 2017;109(11):1-9.

56. Ash DV Waiting times for cancer treatment. Clin Oncol (R Coll Radiol). 2000;12(3):140. [PubMed: 10942327]

57. Mackillop WJ, Fu H, Quirt CF, et al. Waiting for radiotherapy in Ontario. Int J Radiat Oncol Biol Phys. 1994;30(1):221-228. [PubMed: 8083117]

58. Dwyer P, Hickey B, Burmeister E, et al. Hypofractionated whole-breast radiotherapy: impact on departmental waiting times and cost. J Med Imaging Radiat Oncol. 2010;54(3):229-234. [PubMed: 20598011]

59. Bhattacharyya T, Mahajan R, Ghoshal S, et al. Hypofractionated radiotherapy in carcinoma breast: What we have achieved? J Cancer Res Ther. 2015;11(2):259-263. [PubMed: 26148580]

60. Kummerow KL, Du L, Penson DF, et al. Nationwide Trends in Mastectomy for Early-Stage Breast Cancer. JAMA Surg. 2015;150(1):9-16. [PubMed: 25408966]

61. Chu QD, Caldito G, Miller JK, et al. Postmastectomy Radiation for N2/N3 Breast Cancer: Factors Associated with Low Compliance Rate. J Am Coll Surg. 2015;220(4):659-669. [PubMed: 25797752]

62. Showalter SL, Grover S, Sharma S, et al. Factors influencing surgical and adjuvant therapy in stage I breast cancer: a SEER 18 database analysis. Ann Surg Oncol. 2013;20(4):1287-1294. [PubMed: 23135311]

63. Nattinger AB, Kneusel RT, Hoffmann RG, et al. Relationship of distance from a radiotherapy facility and initial breast cancer treatment. J Natl Cancer Inst. 2001;93(17):1344-1346. [PubMed: 11535710]

64. Ragaz J, Olivotto IA, Spinelli JJ, et al. Locoregional Radiation Therapy in Patients With High-Risk Breast Cancer Receiving Adjuvant Chemotherapy: 20-Year Results of the British Columbia Randomized Trial. J Natl Cancer Inst. 2005;97(2):116-126. [PubMed: 15657341]

65. Overgaard M, Nielsen HM, Overgaard J. Is the benefit of postmastectomy irradiation limited to patients with four or more positive nodes, as recommended in international consensus reports? A subgroup analysis of the DBCG 82 b\&c randomized trials. Radiother Oncol. 2007;82(3):247-253. [PubMed: 17306393]

66. Early Breast Cancer Trialists' Collaborative Group (EBCTCG). Effect of radiotherapy after mastectomy and axillary surgery on 10-year recurrence and 20-year breast cancer mortality: metaanalysis of individual patient data for 8135 women in 22 randomised trials. Lancet. 2014;383(9935):2127-2135. [PubMed: 24656685] 
67. Ohri N, Moshier E, Ho A, et al. Postmastectomy Radiation in Breast Cancer Patients With Pathologically Positive Lymph Nodes After Neoadjuvant Chemotherapy: Usage Rates and Survival Trends. Int J Radiat Oncol. 2017;99(3):549-559.

68. Frasier LL, Holden S, Holden T, et al. Temporal Trends in Postmastectomy Radiation Therapy and Breast Reconstruction Associated With Changes in National Comprehensive Cancer Network Guidelines. JAMA Oncol. 2016;2(1):95-101. [PubMed: 26539936]

69. Johansson S, Svensson H, Larsson LG, et al. Brachial plexopathy after postoperative radiotherapy of breast cancer patients--a long-term follow-up. Acta Oncol. 2000;39(3):373-382. [PubMed: 10987234]

70. Johansson S, Svensson H, Denekamp J. Dose response and latency for radiation-induced fibrosis, edema, and neuropathy in breast cancer patients. Int J Radiat Oncol Biol Phys. 2002;52(5):12071219. [PubMed: 11955731]

71. Overgaard M, Bentzen SM, Christensen JJ, et al. The value of the NSD formula in equation of acute and late radiation complications in normal tissue following 2 and 5 fractions per week in breast cancer patients treated with postmastectomy irradiation. Radiother Oncol. 1987;9(1):1-11. [PubMed: 3602425]

72. Jagsi R, Jiang J, Momoh AO, et al. Complications After Mastectomy and Immediate Breast Reconstruction for Breast Cancer: A Claims-Based Analysis. Ann Surg. 2016;263(2):219-227. [PubMed: 25876011]

73. Alderman AK, Wilkins EG, Kim HM, et al. Complications in postmastectomy breast reconstruction: two-year results of the Michigan Breast Reconstruction Outcome Study. Plast Reconstr Surg. 2002;109(7):2265-2274. [PubMed: 12045548]

74. Khan AJ, Poppe MM, Goyal S, et al. Hypofractionated Postmastectomy Radiation Therapy Is Safe and Effective: First Results From a Prospective Phase II Trial. J Clin Oncol. 2017;35(18):20372043. [PubMed: 28459606] * First trial to report safety and efficacy of hypofractionated treatment in the post-mastectomy setting with breast reconstruction and regional nodal irradiation.

75. Shin SM, No HS, Vega RM, et al. Breast, chest wall, and nodal irradiation with prone set-up: Results of a hypofractionated trial with a median follow-up of 35 months. Pract Radiat Oncol. 2016;6(4):e81-88. [PubMed: 26723552]

76. Hypofractionated Radiation Therapy After Mastectomy in Preventing Recurrence in Patients With Stage IIa-IIIa Breast Cancer. National Institute of Health Accessed 2018 Feb 27 Available from: https://clinicaltrials.gov/show/NCT03414970

77. Sun GY, Wang SL, Song YW, et al. Hypofractionated Radiation Therapy After Mastectomy for the Treatment of High-Risk Breast Cancer: 5-Year Follow-Up Result of a Randomized Trial. Int J Radiat Oncol Biol Phys. 2017;99(2):S3-4.

78. FAST-Forward. The Institute of Cancer Research Accessed 2018 Feb 28 Available from: https:// www.icr.ac.uk/our-research/our-research-centres/clinical-trials-and-statistics-unit/clinical-trials/ fast_forward_page

79. Ahlawat S, Haffty BG, Goyal S, et al. Short-Course Hypofractionated Radiation Therapy With Boost in Women With Stages 0 to IIIa Breast Cancer: A Phase 2 Trial. Int J Radiat Oncol Biol Phys. 2016;94(1):118-125. [PubMed: 26700706]

80. Cooper BT, Formenti-Ujlaki GF, Li X, et al. Prospective Randomized Trial of Prone Accelerated Intensity Modulated Breast Radiation Therapy With a Daily Versus Weekly Boost to the Tumor Bed. Int J Radiat Oncol Biol Phys. 2016;95(2):571-578. [PubMed: 27131077]

81. Higher Per Daily Treatment-Dose Radiation Therapy or Standard Per Daily Treatment Radiation Therapy in Treating Patients With Early-Stage Breast Cancer That Was Removed by Surgery. National Institute of Health Accessed 2018 Feb 27 Available from: https://clinicaltrials.gov/ct2/ show/NCT01349322 


\section{Key issues}

- The standard of care for early-stage breast cancer is breast conservation therapy, consisting of breast-conserving surgery followed by adjuvant radiation treatment.

- Conventionally-fractionated whole breast irradiation was generally used, consisting of $50 \mathrm{~Gy}$ in 25 fractions with or without a tumor bed boost, delivered over the course of five to seven weeks, however hypofractionated whole breast irradiation reduces treatment time to three to four weeks resulting in shorter, more convenient, and less costly treatment.

- $\quad$ Large prospective, randomized phase III trials have demonstrated that hypofractionated treatment results in equivalent tumor controls, better or improved acute and late toxicity, and better or improved breast cosmesis compared to conventionally-fractionated regimens for early-stage breast cancer.

- Hypofractionated whole breast irradiation has become the new standard of care for breast conservation therapy; preferred regimens are $40 \mathrm{~Gy}$ in 15 fractions or $42.5 \mathrm{~Gy}$ in 16 fractions with or without a tumor bed boost.

- Hypofractionated whole breast irradiation is also used for ductal carcinoma in-situ on the basis of smaller retrospective studies and extrapolation from randomized studies of invasive cancer.

- $\quad$ Randomized trials are ongoing to assess efficacy and safety of hypofractionated treatment in locally-advanced breast cancers that require regional nodal treatment; early data support its use however broad adoption awaits long-term results.

- Hypofractionation in breast cancer is considered cost-effective and resourceful care that aligns within the overall goals of the healthcare system. 
Table 1.

Key Characteristics and Results of Major Randomized Trials Comparing Hypofractionated and Conventionally-Fractionated Whole Breast Irradiation Regimens

\begin{tabular}{|c|c|c|c|c|c|c|c|c|}
\hline & \multicolumn{2}{|c|}{$\underset{\mathbf{N}=\mathbf{1 , 4 1 0}}{\mathrm{RMH} / \mathrm{GOC}[\mathbf{7 , 8}]}$} & \multicolumn{2}{|c|}{$\begin{array}{l}\text { OCOG }[9,10] \\
N=1,234\end{array}$} & \multicolumn{2}{|c|}{$\begin{array}{l}\text { START A }[11,12] \\
\quad \mathrm{N}=\mathbf{2 , 2 3 6}\end{array}$} & \multicolumn{2}{|c|}{$\begin{array}{l}\text { START B }[12,13] \\
\qquad \mathbf{N}=\mathbf{2 , 2 1 5}\end{array}$} \\
\hline Stage eligibility & \multicolumn{2}{|c|}{ T1-3 N0-1 M0 } & \multicolumn{2}{|c|}{ T1-2 N0 M0 } & \multicolumn{2}{|c|}{ T1-3a N0-1 M0 } & \multicolumn{2}{|c|}{ T1-3a N0-1 M0 } \\
\hline Median follow-up & \multicolumn{2}{|c|}{9.7 years } & \multicolumn{2}{|c|}{12.0 years } & \multicolumn{2}{|c|}{9.3 years } & \multicolumn{2}{|c|}{9.9 years } \\
\hline Age $<50, \mathrm{~N}(\%)$ & \multicolumn{2}{|c|}{$423(30 \%)$} & \multicolumn{2}{|c|}{$305(26 \%)$} & \multicolumn{2}{|c|}{$509(23 \%)$} & \multicolumn{2}{|c|}{$457(21 \%)$} \\
\hline Mastectomy, N (\%) & \multicolumn{2}{|c|}{0} & \multicolumn{2}{|c|}{0} & \multicolumn{2}{|c|}{$336(15 \%)$} & \multicolumn{2}{|c|}{$177(8 \%)$} \\
\hline Chemotherapy, N (\%) & \multicolumn{2}{|c|}{$196(14 \%)$} & \multicolumn{2}{|c|}{$136(11 \%)$} & \multicolumn{2}{|c|}{$793(35 \%)$} & \multicolumn{2}{|c|}{$491(22 \%)$} \\
\hline Boost, N (\%) & \multicolumn{2}{|c|}{$1051(75 \%)$} & \multicolumn{2}{|c|}{0} & \multicolumn{2}{|c|}{$1,152(61 \%)$} & \multicolumn{2}{|c|}{$875(43 \%)$} \\
\hline Regional nodal irradiation, $\mathrm{N}(\%)$ & \multicolumn{2}{|c|}{$290(21 \%)$} & \multicolumn{2}{|c|}{0} & \multicolumn{2}{|c|}{$318(14 \%)$} & \multicolumn{2}{|c|}{$161(7 \%)$} \\
\hline \multirow{3}{*}{$\begin{array}{l}\text { Treatment arms (Gy/fractions) with 10-year local failure } \\
\text { rates }\end{array}$} & $50 / 25$ & $12.1 \%$ & $50 / 25$ & $7.5 \%$ & $50 / 25$ & $6.7 \%$ & $50 / 25$ & $5.2 \%$ \\
\hline & $42.9 / 13$ & $9.6 \%$ & $42.6 / 16$ & $7.4 \%$ & $41.6 / 13$ & $5.6 \%$ & $40 / 15$ & $3.8 \%$ \\
\hline & $39 / 13$ & $14.8 \%$ & & & $39 / 13$ & $8.1 \%$ & & \\
\hline
\end{tabular}

RMH/GOC = Royal Marsden Hospital/Gloucestershire Oncology Centre; OCOG = Ontario Clinical Oncology Group; START = Standardization of Breast Radiotherapy Trial 